\title{
Ropivacaine inhibits cervical cancer cell growth via suppression of the miR-96/MEG2/pSTAT3 axis
}

\author{
XI CHEN ${ }^{1}$, WENXING LIU ${ }^{1}$, XIAOHUA GUO ${ }^{1}$, SHENJIAO HUANG $^{2}$ and XINGRONG SONG ${ }^{1}$ \\ Departments of ${ }^{1}$ Anesthesia and ${ }^{2}$ Gynaecology, Guangzhou Women and \\ Children's Medical Center, Guangzhou Medical University, Guangzhou, Guangdong 510623, P.R. China
}

Received July 7, 2019; Accepted December 9, 2019

DOI: $10.3892 /$ or.2020.7521

\begin{abstract}
Ropivacaine, one of the most commonly used local anesthetics in clinical practice, has shown potent antitumor activity in multiple types of cancer cells. However, its effect on cervical cancer cell growth remains unknown. In the present study, it was found that ropivacaine inhibited cervical cancer cell growth by suppressing cell cycle progression and promoting cell apoptosis, as determined by CCK-8 assay, cell cycle and apoptosis analyses. Western blot analysis and luciferase assay demonstrated that ropivacaine abrogated the phosphorylation and transcriptional activation of signal transducer and activator of transcription 3 (STAT3), and that STAT-3C overexpression reversed the inhibition of cervical cancer cell viability mediated by ropivacaine. Furthermore, our results revealed that the increased expression of maternally expressed gene 2 (MEG2) caused by ropivacaine led to STAT3 dephosphorylation. Finally, we found that ropivacaine upregulated $M E G 2$ by decreasing the expression of microRNA-96 (miR-96). Taken together, our results describe a novel mechanism for the anticancer activity of ropivacaine and suggest ropivacaine as a potential therapeutic agent for cervical cancer patients.
\end{abstract}

\section{Introduction}

Signal transducer and activator of transcription 3 (STAT3) is the vital member of the STAT family, which participates in various cellular processes such as proliferation, apoptosis, migration and differentiation (1). In response to multiple

Correspondence to: Dr Xingrong Song, Department of Anesthesia, Guangzhou Women and Children's Medical Center, Guangzhou Medical University, 9 Jinhui Road, Guangzhou, Guangdong 510623, P.R. China

E-mail: songxr1966@163.com

Abbreviations: STAT3, signal transducer and activator of transcription 3; JAK, Janus associated kinase; PTPs, protein tyrosine phosphatases; $M E G 2$, maternally expressed gene 2

Key words: ropivacaine, cervical cancer, miR96, MEG2, STAT3 extracellular stimuli that include diverse growth factors as well as cytokines, cytoplasmic STAT3 becomes phosphorylated at the residue Tyr705 by Janus-associated kinase (JAK), forms homo- or heterodimers and translocates to the nucleus. In the nucleus, STAT3 activates transcription of target genes by binding to their promoters (2). Due to its importance in cellular processes, STAT3 phosphorylation is tightly regulated. Phosphorylated STAT3 can be dephosphorylated by multiple protein tyrosine phosphatases (PTPs), including PTR receptor type D (PTPRD), PTR receptor type T (PTPRT), Src homology region 2 domain-containing phosphatase-1 (SHP1), Src homology region 2 domain-containing phosphatase-2 (SHP2) or maternally expressed gene $2(M E G 2)$, resulting in its inactivation (3).

Cervical cancer is the second most commonly diagnosed malignant tumor in women worldwide and the third-leading cause of cancer-related deaths among women in less-developed countries (4). Despite therapeutic advances in recent years, the mortality and relapse rates of cervical cancer remain high. Therefore, it is urgent to develop novel effective therapeutic approaches to improve the outcome of cervical cancer treatment. STAT3 has been reported to be abnormally activated in cervical cancer, and a high level of STAT3 phosphorylation predicts poor clinical prognosis (5-7). Furthermore, aberrant activation of STAT3 has been shown to promote the growth of cervical cancer cells (8). All these findings suggest that STAT3 is a promising molecular target for the treatment of cervical cancer.

Ropivacaine, a voltage-gated sodium channel inhibitor, is widely used as a local anesthetic to relieve pain in clinical practice (9). Apart from the anesthetic advantages, increasing clinical evidence demonstrates that the use of local anesthetics during cancer surgery may decrease the risk of recurrence and metastasis $(10,11)$. In addition, recent studies show that ropivacaine exhibits anticancer properties in multiple types of cancers, including leukemia (12), hepatocellular carcinoma (13), colon cancer (14), pancreatic cancer (14), gastric cancer (15), esophageal cancer (16) and lung cancer (17) by diverse molecular mechanisms. However, whether and how ropivacaine suppresses cervical cancer cell growth remains unknown. In the present study, ropivacaine was found to exert an inhibitory effect on the viability of cervical cancer cells by suppressing cell cycle progression and promoting cell apoptosis. Mechanistically, ropivacaine inhibited 
cervical cancer cell growth by targeting the microRNA-96 (miR-96)/MEG2/pSTAT3 axis.

\section{Materials and methods}

Cell lines and cell culture. The human cervical cancer cell lines Siha and Caski were obtained from Fengh Bio. Inc. (Changsha, China) and EK-Bioscience, Inc. (Shanghai, China), respectively. The cells were maintained in Dulbecco's modified Eagle's medium (DMEM; HyClone, Thermo Fisher Scientific, Inc.) supplemented with $10 \%$ fetal bovine serum (FBS) (Gibco, Life Technologies Inc., Thermo Fisher Scientific, Inc.) and 1\% penicillin/streptomycin (Sigma-Aldrich; Merck $\mathrm{KGaA}$ ) in a humidified incubator with $5 \% \mathrm{CO}_{2}$ at $37^{\circ} \mathrm{C}$.

For stable overexpression of STAT-3C, SiHa cells were transfected with the pCMV-2B-STAT-3C vector using Lipofectamine $^{\mathrm{TM}} 2000$ (Invitrogen; Thermo Fisher Scientific, Inc.) following the manufacturer's protocol. SiHa cells transfected with the pCMV-2B backbone were used as control cells. Stable transformants were selected with $800 \mu \mathrm{g} / \mathrm{ml} \mathrm{G} 418$ (Calbiochem) for two weeks before subsequent experimentation.

Antibodies and reagents. STAT3 (cat. no. \#9139, dilution 1:2,000), phospho-STAT3-Tyr705 (cat. no. \#4093, dilution 1:500), cyclin D1 (cat. no. \#2922, dilution 1:2,000), survivin (cat. no. \#2802, dilution 1:1,000), JAK2 (cat. no. \#3230, dilution 1:500) and phospho-JAK2-Tyr1007 (cat. no. \#4406, dilution 1:500) antibodies were purchased from Cell Signaling Technology. PTPRT (cat. no. ab115848, dilution 1:1,000), SHP-1 (cat. no. ab124942, dilution 1:1,000) and SHP-2 (cat. no. ab131541, dilution 1:1,000) antibodies were purchased from Abcam. PTPRD (cat. no. LS-C153706, dilution 1:1,000) antibody was purchased from Life Span BioSciences. $\gamma$-tubulin (cat. no. sc-7396, dilution 1:500) antibody was obtained from Santa Cruz Biotechnology. MEG2 (cat. no. MAB2668, dilution 1:1,000) was purchased from R\&D Systems, Inc. Ropivacaine was obtained from Selleck Chemicals. Pervanadate were purchased from Sigma Aldrich; Merck KGaA.

Western blot analysis. Protein was harvested from cells and the protein concentration was measured using the BCA method (Pierce Chemical; Thermo Fisher Scientific, Inc.). Protein $(50 \mu \mathrm{g})$ was resolved by $10 \%$ sodium dodecyl sulphate-polyacrylamide (SDS-PAGE) gel electrophoresis and transferred to a nitrocellulose membrane. After being blocked with 5\% nonfat milk in TBST for $2 \mathrm{~h}$ at room temperature, the membrane was incubated with the primary antibody at $4^{\circ} \mathrm{C}$ overnight, followed by incubation with the corresponding HRP-conjugated secondary antibody for $2 \mathrm{~h}$ at room temperature. After being incubated with freshly prepared chemiluminescence solution for 1-5 min, the membrane was observed using enhanced chemiluminescence (ECL) detection reagent (Thermo Fisher Scientific, Inc.) in an ImageQuant LAS4000 chemiluminescence imager (GE Healthcare Life Sciences). Image Lab version 3.0 software (Bio-Lab) was used to perform the densitometric analysis of blots. Tubulin was used as the loading control.

Nuclear fraction isolation. The nuclear proteins of $\mathrm{SiHa}$ or Caski cells were extracted using the NE-PER Nuclear and
Cytoplasmic Extraction Reagents (Thermo Fisher Scientific, Inc.) according to the manufacturer's instructions. Briefly, after being washed with ice-cold PBS and centrifuged at $500 \mathrm{x} \mathrm{g}$ at $4^{\circ} \mathrm{C}$ for $3 \mathrm{~min}$, the cells was treated with CER I, shaken vigorously for $15-30 \mathrm{sec}$ and incubated at $4^{\circ} \mathrm{C}$ for $10 \mathrm{~min}$. Then, CER II was added into the lysis, followed by vigorous vortexing for $10 \mathrm{sec}$ and incubation on ice for $1 \mathrm{~min}$. After centrifugation at $16,000 \mathrm{x}$ g at $4^{\circ} \mathrm{C}$ for $5 \mathrm{~min}$, nuclear extraction reagent was added to the pellet followed by incubation at $4^{\circ} \mathrm{C}$ for $40 \mathrm{~min}$ with occasional vortexing. The nuclear fraction was finally harvested following centrifugation at $16,000 \mathrm{x}$ g at $4^{\circ} \mathrm{C}$ for $10 \mathrm{~min}$.

Luciferase assay. SiHa and Caski cells were seeded in 24-well plates at a density of $2 \times 10^{4}$ cells/per well. On the second day, the cells was co-transfected with $200 \mathrm{ng}$ of pAPRE-luc reporter plasmid and $20 \mathrm{ng}$ pRL-TK plasmid as an internal control. After $24 \mathrm{~h}$, the transferred cells were treated with different concentrations $(0,0.25,0.5,1 \mathrm{mM})$ of ropivacaine for $72 \mathrm{~h}$. Then, the cells were collected for luciferase activity measurement with the Dual-Luciferase Reporter Assay system (Promega) according to the manufacturer's instructions.

CCK-8 assay. SiHa or Caski cells were plated into 96-well plates at a density of 2,000 cells per well. On the following day, the cells were treated with different concentrations $(0,0.25,0.5$, $1 \mathrm{mM}$ ) of ropivacaine for $72 \mathrm{~h}$. Then, the viability of the cells was detected by measurement of absorbance at $450 \mathrm{~nm}$ with the Cell Counting Kit-8 (CCK-8) (Dojindo Laboratories) assay following the manufacturer's instructions. The experimental optical density (OD) value was normalized to the control OD value. Assays were performed in triplicate, and the results are presented as means \pm standard deviation (SD).

BrdU incorporationassay. SiHa orCaski cells (2,000 cells/well) were plated into 96-well plates. On the second day, the cells were treated with the indicated concentrations $(0,0.25,0.5$, $1 \mathrm{mM}$ ) of ropivacaine for $72 \mathrm{~h}$. Then, the cells were further incubated in culture medium with $10 \mu \mathrm{M} \mathrm{BrdU}$ for an additional $10 \mathrm{~h}$. Subsequently, the cells were fixed, and BrdU incorporation was determined using a BrdU Cell Proliferation ELISA kit (cat. no. 11647229001, Roche Applied Science) following the manufacturer's protocol. The BrdU density was analyzed by measurement of absorbance at $450 \mathrm{~nm}$. The experiments were performed in triplicate and the results are presented as means \pm standard deviation (SD).

Colony formation assay. SiHa and Caski cells were seeded into 6-well plates at a density of 2,000 cells per well. Cells were allowed to grow in DMEM/10\% FBS medium with different concentrations $(0,0.25,0.5,1 \mathrm{mM})$ of ropivacaine for $72 \mathrm{~h}$ for 2 weeks. After being fixed with ice-cold methanol and stained with $0.5 \%$ crystal violet solution for $20 \mathrm{~min}$ at room temperature, the colony numbers were calculated using Image J software, version 1.49 [National Institutes of Health (NIH), Bethesda, MD, USA]. Assays were performed in triplicate, and the results are presented as means \pm standard deviation (SD).

Cell cycle analysis. SiHa and Caski cells $\left(1 \times 10^{5}\right.$ cells/well) were seeded into a 6-well plate. The following day, the cells were treated with different concentrations $(0,0.25,0.5,1 \mathrm{mM})$ of 
ropivacaine for $72 \mathrm{~h}$ and then collected by trypsinization. After being washed twice with ice-cold phosphate-buffered saline (PBS), the cells were fixed with $70 \% \mathrm{EtOH}$ overnight. After incubation with $200 \mu \mathrm{g} / \mathrm{ml}$ DNase-free RNase A for $30 \mathrm{~min}$ at $37^{\circ} \mathrm{C}$, the cells were stained with $0.05 \mathrm{mg} / \mathrm{ml}$ PI for $15 \mathrm{~min}$ in the dark at room temperature, followed by flow cytometric analysis.

Cell apoptosis analysis. SiHa and Caski cells ( $1 \times 10^{5}$ cells/well) were seeded into a 6 -well plate. After $24 \mathrm{~h}$, the cells were treated with different concentrations $(0,0.25,0.5,1 \mathrm{mM})$ of ropivacaine for $72 \mathrm{~h}$ and then harvested by trypsinization. The cells were washed twice with ice-cold PBS, and then stained with Annexin V and propidium iodide (PI) using an Annexin V-FITC Apoptosis Detection Kit (BD Biosciences). Apoptosis was measured by flow cytometric analysis according to the manufacturer's protocol. Data were analyzed using FlowJo software version 10 (Tree Star, Inc.).

siRNA transfections. siRNA for MEG2 was purchased from Invitrogen; Thermo Fisher Scientific, Inc. SiHa cells were transfected with $M E G 2$ siRNA or NC siRNA with Lipofectamine RNAiMAX (Invitrogen; Thermo Fisher Scientific, Inc.) following the manufacturer's instructions.

Transient transfection of miR-96 mimics. miR-96 mimics (Ribo Company) were transfected into SiHa cells using Lipofectamine RNAiMAX Transfection Reagent (Invitrogen; Thermo Fisher Scientific, Inc.) according to the manufacturer's instructions. At $18 \mathrm{~h}$ post-transfection, the cells were used for subsequent experiments.

Quantitative real-time PCR. miRNA was isolated with a mirVana miRNA Isolation Kit (Ambion) according to the manufacturer's protocols. Expression of miRNAs was measured with the PrimeScript miRNA RT-PCR kit (Takara) following the manufacturer's instructions. U6 was used as an endogenous control. The forward primer for miR-24 was 5'-TGGCTCAGT TCAGCAGGAACAG-3'. The forward primer for miR-96 was 5'-TTTGGCACTAGCACATTTTTG CT-3'. The forward primer for miR-126 was 5'-TCGTACCGT GAGTAATAATGCG-3'. The forward primer for miR-181a-5p was 5'-AACATTCAACGCTGTCGGTGAGT-3'. The forward primer for miR-613 was 5'-AGGAATGTTCCTTCTTTGCC-3' and the reverse primer for miRNAs was the UnimiRqPCR Primer (Takara). The forward primer for U6 was 5'-ATTGGA ACGATACAGAGAAGATT-3' and the reverse primer for U6 was 5'-GGAACGCTTCACGAATTTG-3'.

Statistical analysis. All data were analyzed using the SPSS 17.0 software (SPSS, Inc.) and are shown as mean \pm standard deviation (SD). The unpaired Student's t-test and one-way ANOVA followed by Dunnett's test were used to determine the statistical significance for comparing two groups and more than two groups, respectively. $\mathrm{P}$-values $<0.05$ were considered statistically significant.

\section{Results}

Ropivacaine inhibits the growth of cervical cancer cells. To explore the effects of ropivacaine on the viability of cervical cancer cells, we treated two cervical cancer cell lines, $\mathrm{SiHa}$ and Caski, with ropivacaine at different concentrations $(0,0.25,0.5$ and $1 \mathrm{mM}$ ) for $72 \mathrm{~h}$, and then measured cell growth using a Cell Counting Kit-8 (CCK-8) assay. The results showed that ropivacaine significantly inhibited the growth of both $\mathrm{SiHa}$ and Caski cell lines in a dose-dependent manner (Fig. 1A and B). Accordingly, it also significantly suppressed incorporation of 5-bromo-2'-deoxyuridine (BrdU) into both $\mathrm{SiHa}$ and Caski cell lines (Fig. 1C and D), confirming its inhibitory effect on the proliferation of cervical cancer cells. Furthermore, the results of colony forming assays indicated that ropivacaine significantly attenuated the survival ability of both SiHa and Caski cell lines (Fig. 1E and F). Together, these data indicated that ropivacaine exhibited a significant inhibitory effect on cervical cancer cell growth.

Ropivacaine suppresses the cell cycle and promotes the apoptosis of cervical cancer cells. Both cell cycle suppression and an increase in cell apoptosis lead to reduced viability in cancer cells. Therefore, we next examined the effect of ropivacaine on the cell cycle and apoptosis of cervical cancer cells. As shown in Fig. 2A, ropivacaine significantly decreased the percentage of cells in the $\mathrm{S}$-phase in a dose-dependent manner. Consistent with these results, cyclin D1 expression was also suppressed by ropivacaine treatment, confirming the drug's inhibitory effect on the cell cycle of cervical cancer cells (Fig. 2B). We next determined the effect of ropivacaine on the apoptosis of cervical cancer cells via an Annexin V apoptosis detection assay. As shown in Fig. 2C, ropivacaine significantly induced accumulation of apoptotic cells in both the SiHa and Caski cell lines in a dose-dependent manner. Accordingly, expression of survivin, a vital anti-apoptosis regulator, was suppressed in a dose-dependent manner as well (Fig. 2D). Taken together, these results indicated that ropivacaine's inhibitory effect on cervical cancer cell growth was mediated by the decrease in cell cycle progression and an increase in apoptosis.

Ropivacaine suppresses phosphorylation and transcriptional activation of STAT3. We next investigated the relevant signaling pathways that mediated ropivacaine's inhibition of cervical cancer cell viability. STAT3 is reported to be abnormally activated in cervical cancers and to contribute to the initiation and development of such cancers by controlling the expression of cell cycle and anti-apoptosis regulators $(7,8)$. Thus, we hypothesized that ropivacaine might attenuate the viability of cervical cancer cells by suppressing STAT3 activation. To test this hypothesis, we first detected the effect of ropivacaine on phosphorylation of STAT3 Tyr705 (pSTAT3-705), which is essential for STAT3 activation. As shown in Fig. 3A, ropivacaine decreased the phosphorylation of STAT3. Since the translocation of STAT3 from the cytoplasm to the nucleus is modulated by its phosphorylation, we next examined whether ropivacaine affected the expression of nuclear STAT3. Consistent with the decrease in STAT3 phosphorylation, nuclear STAT3 expression was markedly inhibited by ropivacaine treatment (Fig. 3B), while the expression of total STAT3 protein was unchanged (Fig. 3A). To further investigate whether the reduction in nuclear STAT3 affected its transcriptional activity, we used an acute-phase response element (APRE) luciferase reporter, which responds 
A
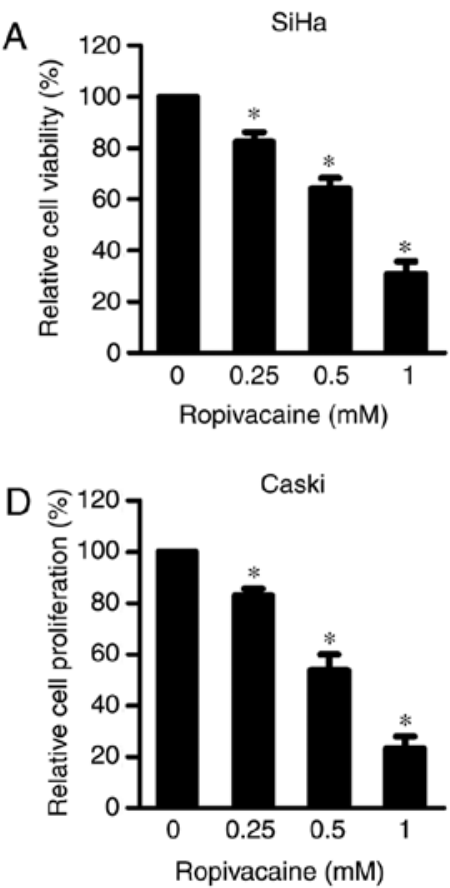

B

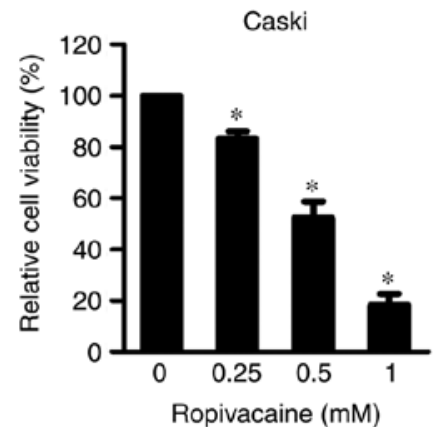

E

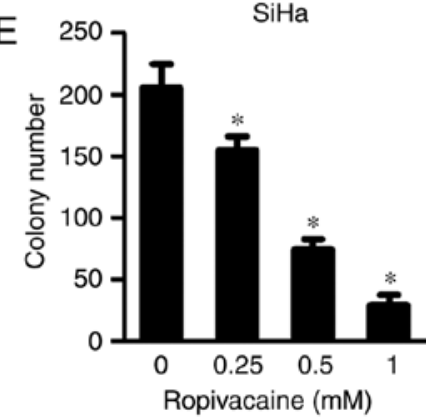

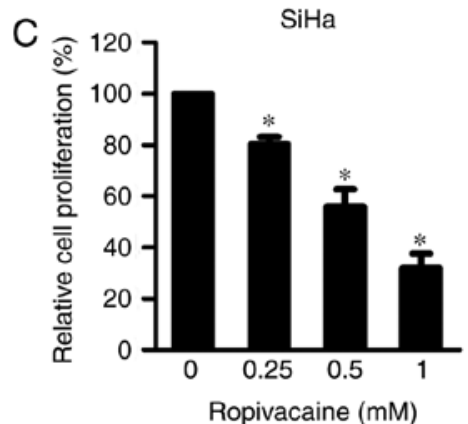

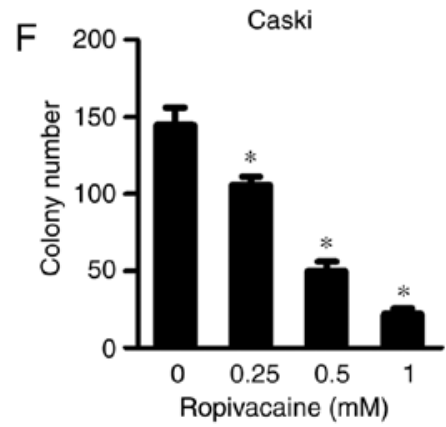

Figure 1. Ropivacaine exhibits an inhibitory effect on the growth of cervical cancer cells. (A) SiHa and (B) Caski cells were treated with indicated concentrations $(0,0.25,0.5$ and $1 \mathrm{mM})$ of ropivacaine. After treatment for $72 \mathrm{~h}$, cell viability was determined using CCK-8 assay. (C) SiHa and (D) Caski cells were treated with the indicated concentrations of ropivacaine for $72 \mathrm{~h}$ and then $\mathrm{BrdU}$ incorporation assay was performed to measure cell proliferation. (E) SiHa and (F) Caski cells were treated with the indicated concentrations of ropivacaine for 2 weeks and then colony forming assay was conducted to evaluate the effect of ropivacaine treatment on cervical cancer cell survival ability. Results are shown as means \pm SD of triplicate measurements. P-values were determined using one-way ANOVA followed by Dunnett's test in comparison with $0 \mathrm{mM}$ ropivacaine-treated cells. ${ }^{*} \mathrm{P}<0.05$.
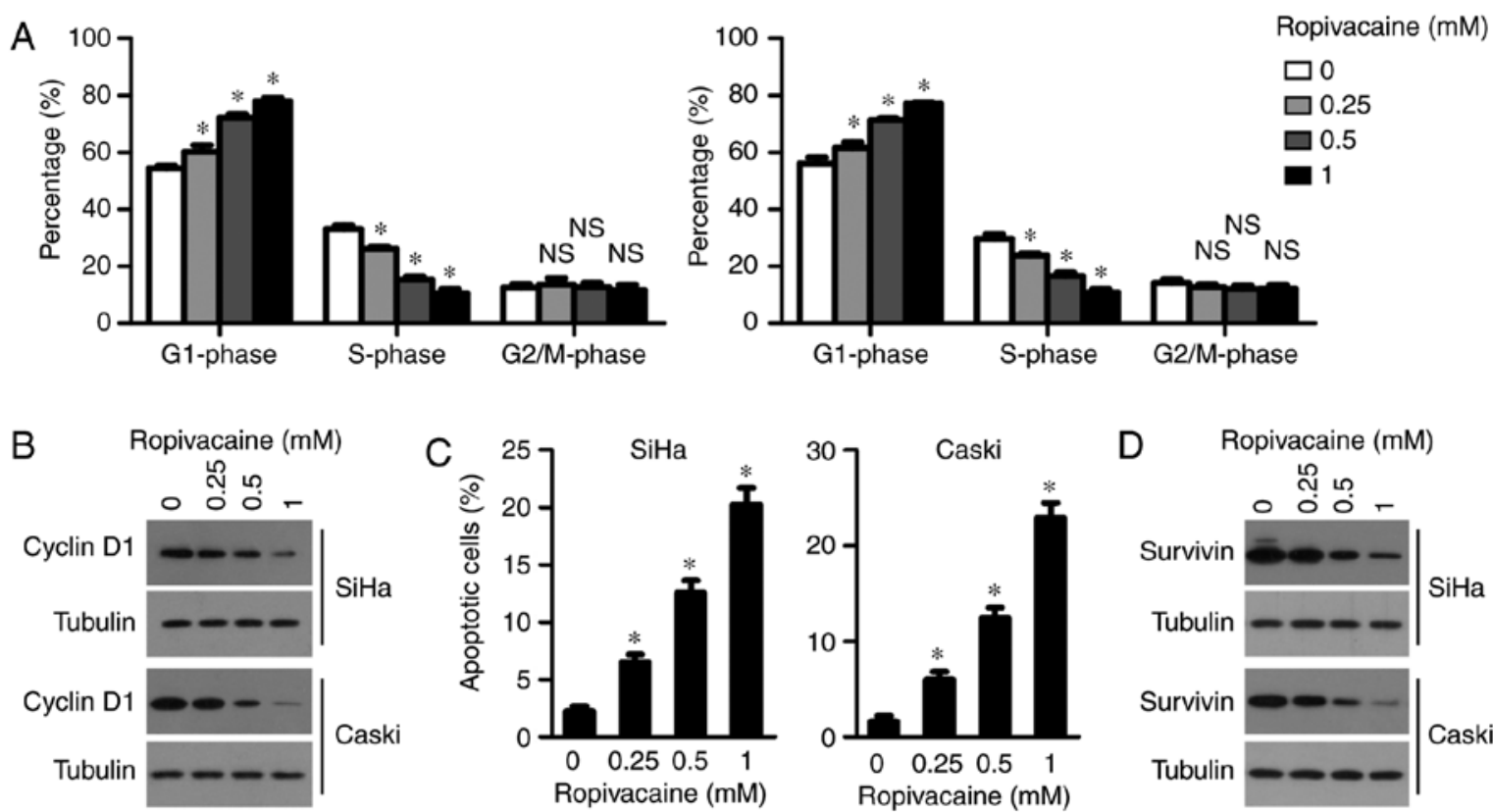

Figure 2. Ropivacaine suppresses the cell cycle and promotes apoptosis of cervical cancer cells. (A) SiHa and Caski cells were treated with indicated concentrations $(0,0.25,0.5$ and $1 \mathrm{mM})$ of ropivacaine for $72 \mathrm{~h}$ and then collected for flow cytometry analysis of cell cycle distribution. (B) Western blot analysis of cyclin D1 expression in SiHa and Caski cells treated with various concentrations of ropivacaine for $72 \mathrm{~h}$. Tubulin was used as a loading control. (C) SiHa and Caski cells were treated with the indicated concentrations of ropivacaine for $72 \mathrm{~h}$ and then Annexin V staining assay was performed to detect cell apoptosis. (D) Western blot analysis of survivin expression in SiHa and Caski cells treated with various concentrations of ropivacaine for $72 \mathrm{~h}$. Tubulin was used as a loading control. Results are shown as means \pm SD of triplicate measurements. P-values were determined using one-way ANOVA followed by Dunnett's test in comparison with $0 \mathrm{mM}$ ropivacaine treated cells. ${ }^{*} \mathrm{P}<0.05$. NS, not significant.

to STAT3 activation. The results showed that ropivacaine led to a significant decrease in luciferase activity in both SiHa and
Caski cell lines (Fig. 3C), indicating that the drug suppressed phosphorylation and transcriptional activation of STAT3. 
A

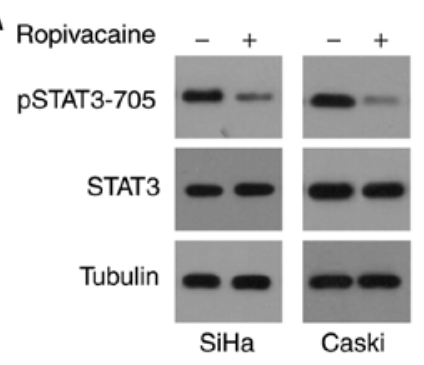

B

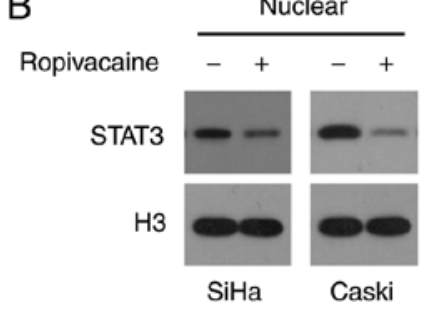

C

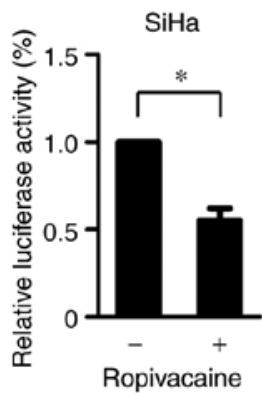

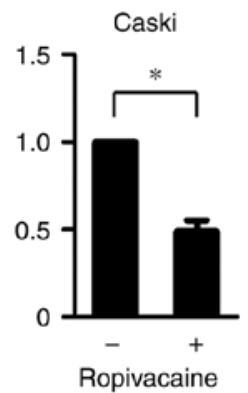

Figure 3. Ropivacaine suppresses the phosphorylation and transcriptional activation of STAT3. (A) After $72 \mathrm{~h}$ of ropivacaine $(0.5 \mathrm{mM})$ treatment, the expression of indicated proteins in SiHa and Caski cells was detected by western blot analysis. Tubulin was used as a loading control. (B) SiHa and Caski cells were treated with ropivacaine $(0.5 \mathrm{mM})$ for $72 \mathrm{~h}$, followed by nuclear protein isolation and western blot analysis of STAT3 expression levels in the nucleus. Histone $\mathrm{H} 3$ was used as a loading control. (C) Luciferase reporter assays. SiHa and Caski cells were transfected with pAPRE-luc reporter plasmid for $24 \mathrm{~h}$ and then cells were treated with ropivacaine $(0.5 \mathrm{mM})$ for $72 \mathrm{~h}$, followed by luciferase activities measurement. Results are shown as means \pm SD of triplicate measurements. The unpaired Student t-test was used to analyze the data. ${ }^{*}<0.05$. STAT3, signal transducer and activator of transcription 3.
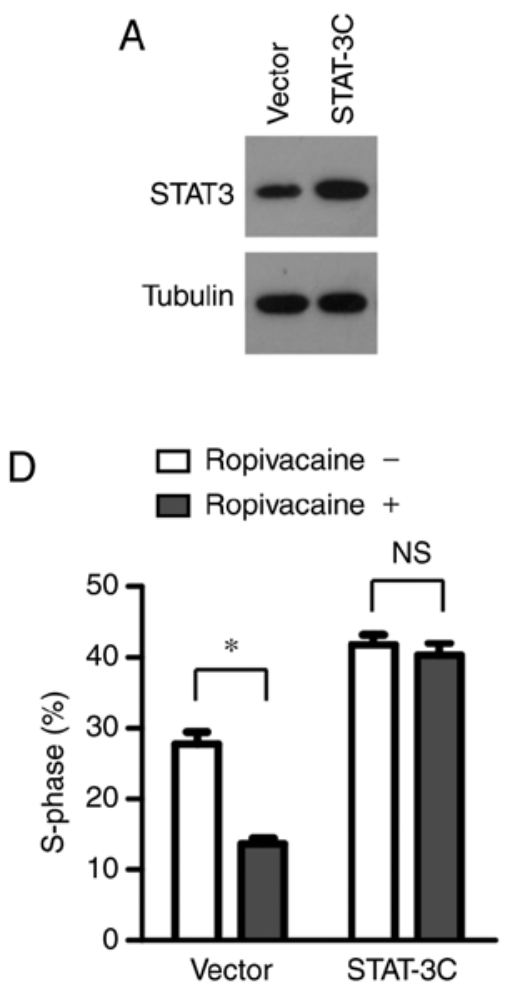

B
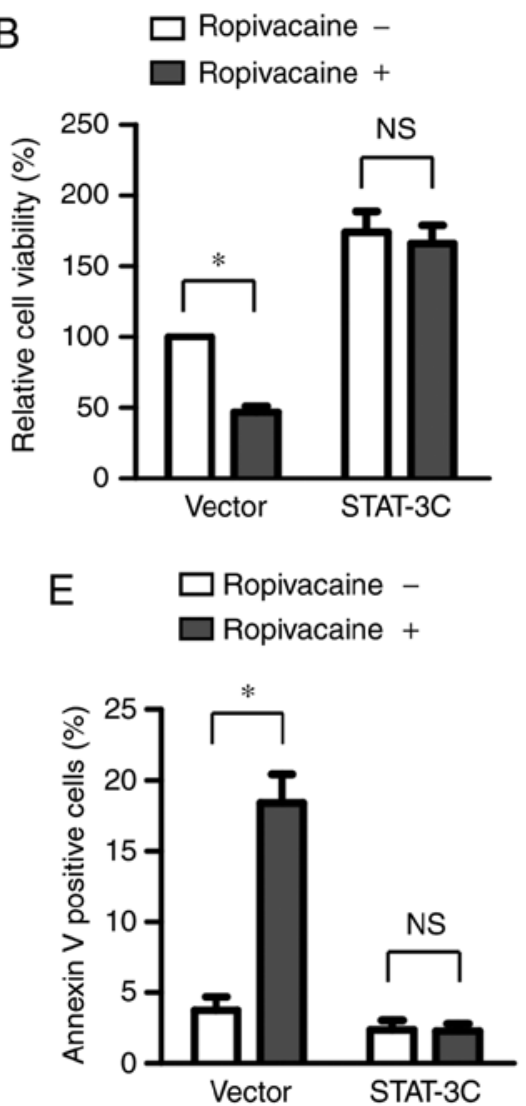
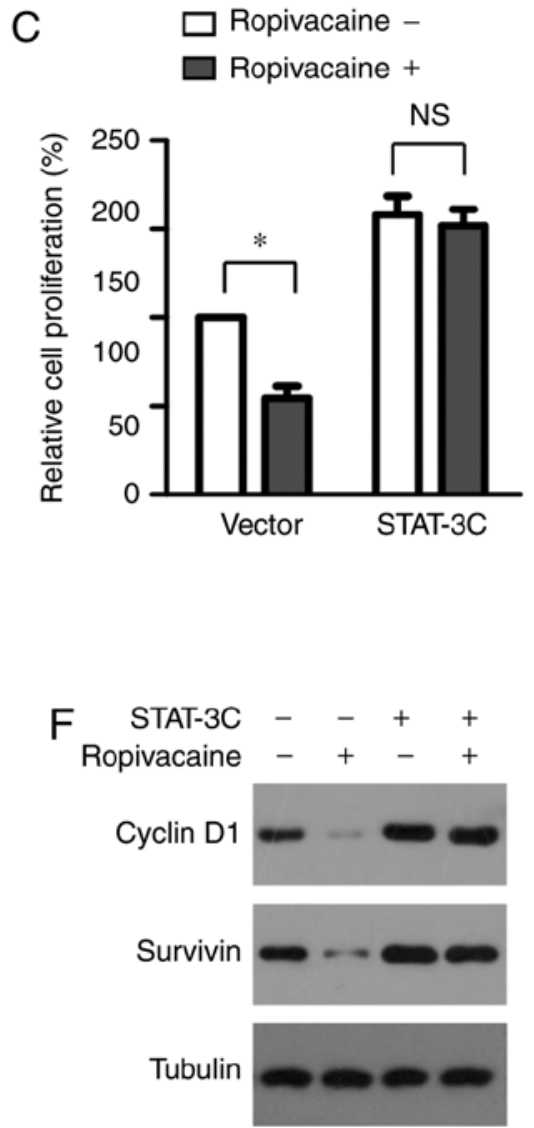

Figure 4. Inhibitory effect of ropivacaine on the growth of cervical cancer cells is dependent on the suppression of STAT3 activation. (A) Successful expression of STAT-3C in SiHa cells with stable expression of STAT-3C, as assessed by western blot analysis. Tubulin was used as a loading control. (B-E) Control and $\mathrm{SiHa}$ cells with stable expression of STAT-3C were treated with ropivacaine $(0.5 \mathrm{mM})$ for $72 \mathrm{~h}$, followed by CCK-8 assay (B), BrdU incorporation assay (C), cell cycle analysis (D) and cell apoptosis analysis (E). (F) Western blot analysis of cyclin D1 and survivin expression in Control and SiHa cells with stable expression of STAT-3C treated with ropivacaine $(0.5 \mathrm{mM})$ for $72 \mathrm{~h}$. Tubulin was used as a loading control. Results are shown as means \pm SD of triplicate measurements. The unpaired Student t-test was used to analyze the data. ${ }^{*} \mathrm{P}<0.05$. NS, not significant; STAT3, signal transducer and activator of transcription 3.

Inhibition of cervical cancer cell growth mediated by ropivacaine is dependent on suppression of STAT3 activation. To test whether the decreased transcriptional activation of STAT3 led to the inhibitory effects mediated by ropivacaine on cervical-cancer cell viability, we established SiHa cells stably expressing STAT-3C (STAT-3C), a constitutive active form of STAT3 (Fig. 4A). We treated both SiHa STAT-3C and
Vector (control cells) transfected cells with $0.5 \mathrm{mM}$ ropivacaine for $72 \mathrm{~h}$ and then performed a CCK-8 assay. Consistent with a previous report (8), STAT-3C overexpression promoted the viability of cervical cancer cells. Interestingly, it also markedly reversed ropivacaine's inhibition of cell viability (Fig. 4B). Additionally, the forced expression of STAT-3C effectively abrogated ropivacaine-mediated suppression of cell 
A
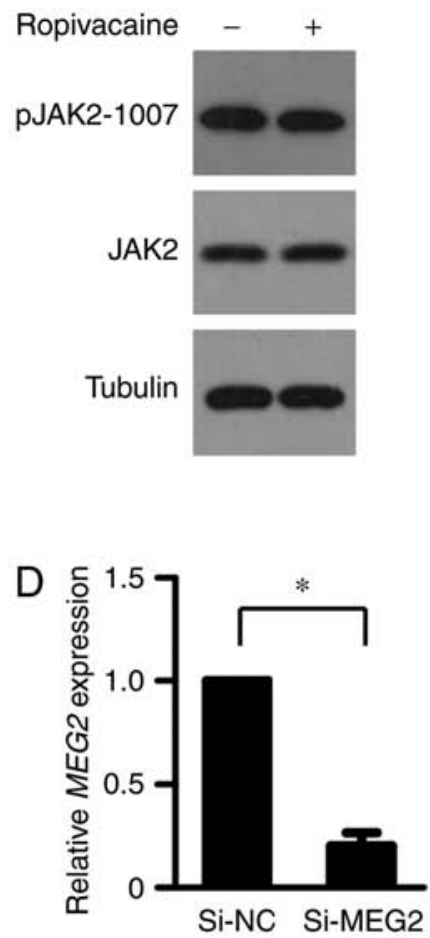

B

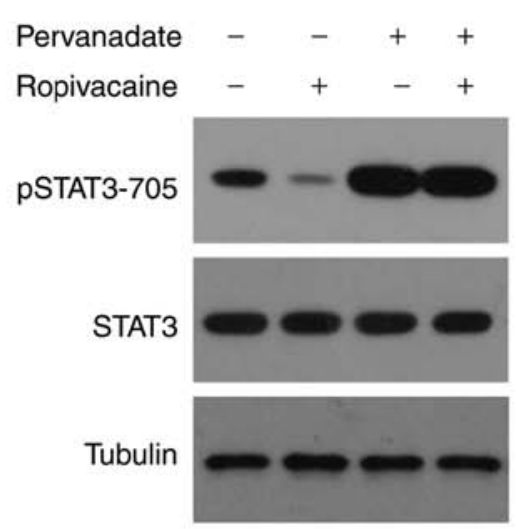

$E$

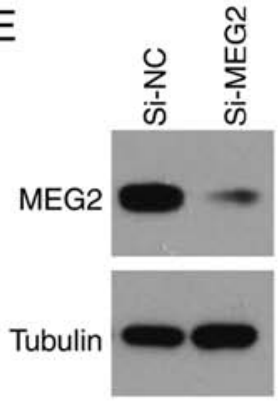

C

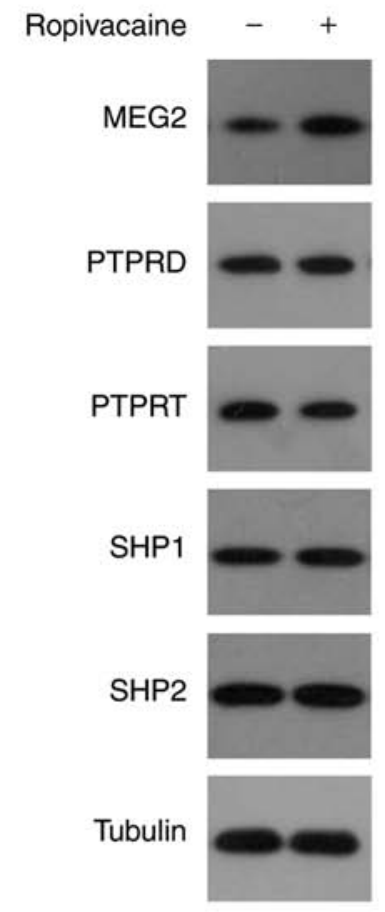

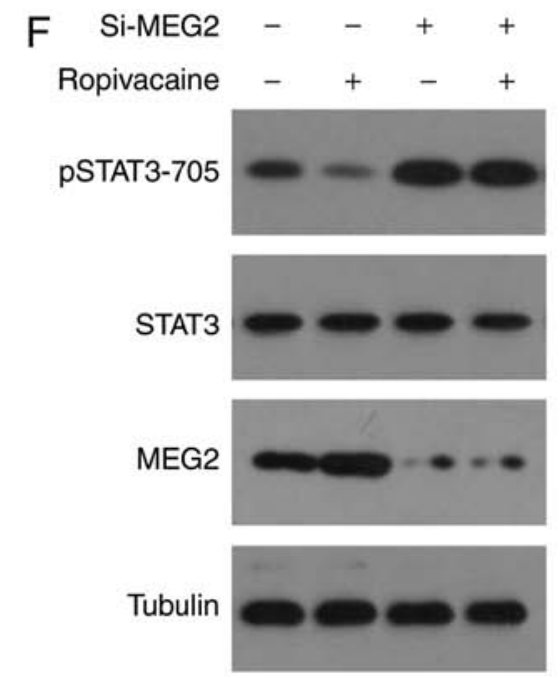

G

Ropivacaine -

Ropivacaine +

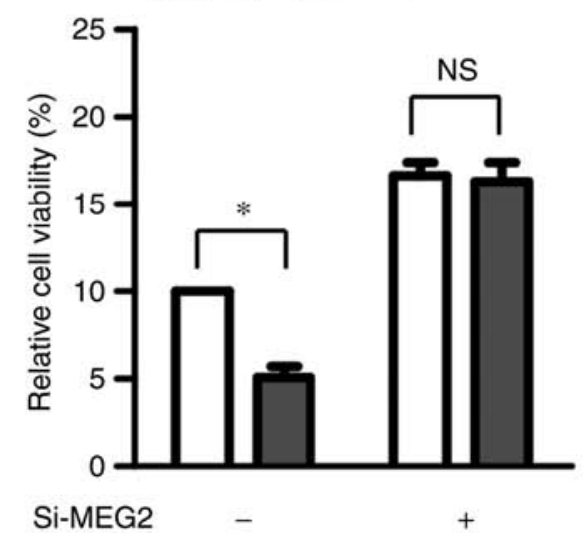

Figure 5. Inhibitory effect of ropivacaine on the phosphorylation of STAT3 is mediated by MEG2. (A) Western blot analysis of pJAK2 levels in SiHa cells treated with ropivacaine $(0.5 \mathrm{mM})$ for $72 \mathrm{~h}$. Tubulin was used as a loading control. (B) SiHa cells were treated with ropivacaine $(0.5 \mathrm{mM})$ and pervanadate $(50 \mu \mathrm{M})$, alone or in combination for $72 \mathrm{~h}$, followed by western blot analysis of pSTAT3 levels. Tubulin was used as a loading control. (C) After $72 \mathrm{~h}$ of ropivacaine $(0.5 \mathrm{mM})$ treatment, the expression levels of indicated proteins in SiHa cells were analyzed by western blot analysis. Tubulin was used as a loading control. (D and E) Successful depletion of MEG2 in SiHa cells transfected with MEG2 siRNA, as assessed by real-time PCR analysis (D) and western blot analysis (E). (F and G) SiHa cells transfected with NC siRNA or MEG2 siRNA were treated with ropivacaine ( $0.5 \mathrm{mM})$ for $72 \mathrm{~h}$, followed by western blot analysis of pSTAT3 levels with tubulin as a loading control (F), and CCK-8 assay (G). Results are shown as means \pm SD of triplicate measurements. The unpaired Student t-test was used to analyze the data. ${ }^{*} \mathrm{P}<0.05$. NS, not significant; pJAK2, phosphorylated Janus associated kinase 2; pSTAT3, phosphorylated signal transducer and activator of transcription 3; $M E G 2$, maternally expressed gene 2; PTPRD, PTR receptor type D; PTPRT, PTR receptor type T (PTPRT); SHP1, Src homology region 2 domain-containing phosphatase-1; SHP2, Src homology region 2 domain-containing phosphatase-2.

proliferation and cell cycle progression, as determined by BrdU incorporation assay and cell cycle analysis (Fig. 4C and D). Furthermore, the increased apoptosis of SiHa cells induced by ropivacaine was rescued by STAT-3C overexpression (Fig. 4E). Accordingly, although we observed reduced expression of cyclin D1 and survivin in the Vector cells in the presence of ropivacaine, we found no significant change in the $\mathrm{SiHa}$
STAT-3C transfected cells (Fig. 4F). Together, these results indicated that ropivacaine attenuated the growth of cervical cancer cells by suppressing STAT3 activation.

MEG2 mediates the inhibition of STAT3 phosphorylation by ropivacaine. To explore the molecular mechanism by which ropivacaine inhibits STAT3 phosphorylation, we first 

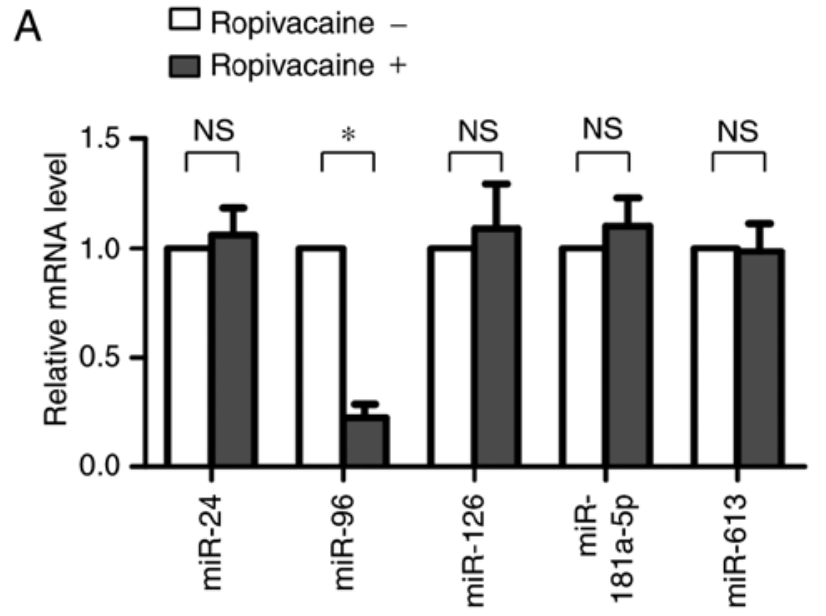

B

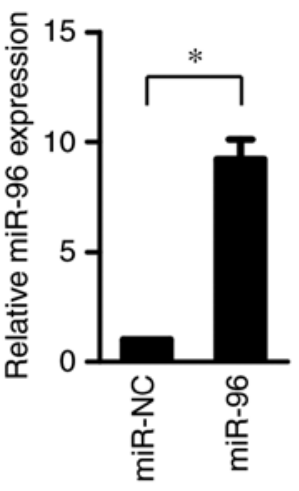

C
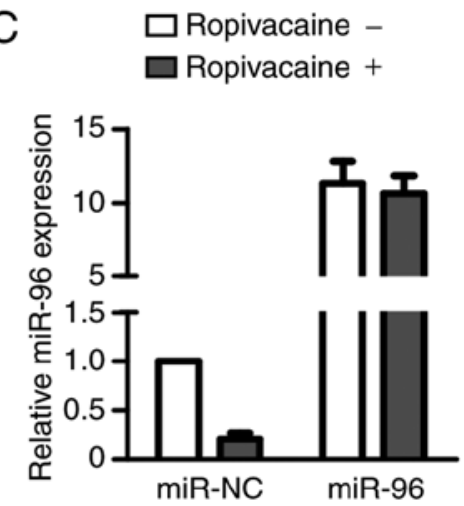
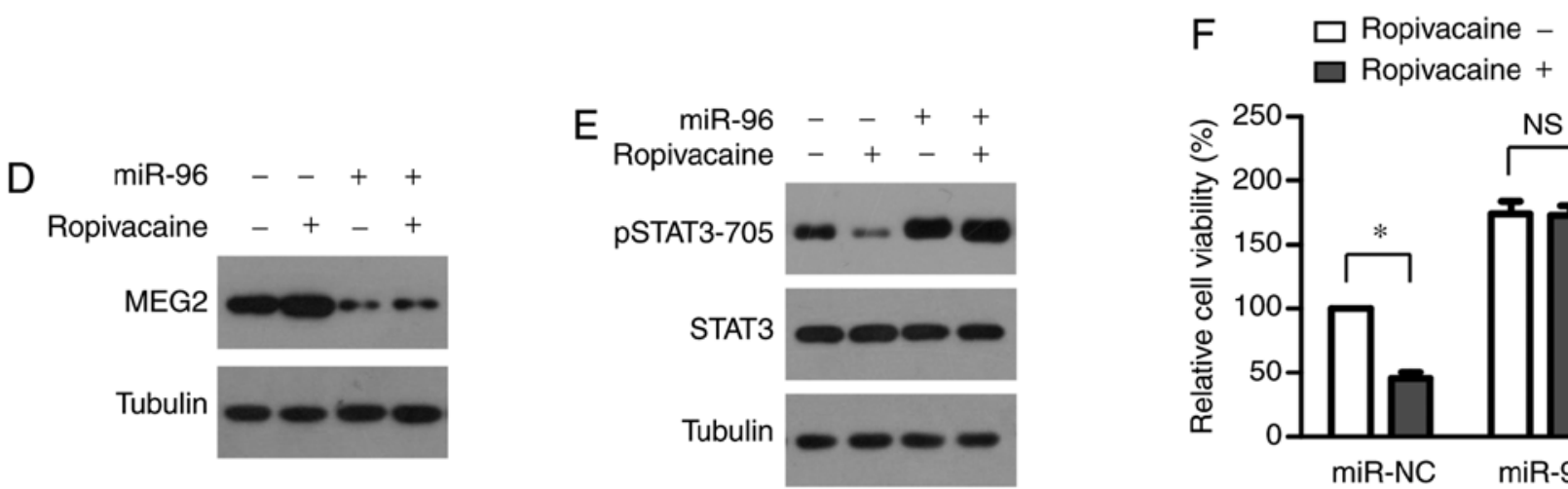

Figure 6. Ropivacaine upregulates MEG2 via suppressing the expression of miR-96. (A) SiHa cells were treated with ropivacaine (0.5 mM) for 72 h, followed by real-time PCR analysis of miR-24, miR-96, miR-126, miR-181a-5p and miR-613. (B) Successful overexpression of miR-96 in SiHa cells transfected with miR-96 mimics, as assessed by real-time PCR analysis. (C) After $72 \mathrm{~h}$ of ropivacaine (0.5 mM) treatment, the expression of miR-96 in SiHa cells transfected with miR-NC or miR-96 mimics were analyzed by real-time PCR analysis. (D) After $72 \mathrm{~h}$ of ropivacaine (0.5 mM) treatment, the expression of MEG2 in SiHa cells transfected with miR-NC or miR-96 mimics were analyzed by western blot analysis. Tubulin was used as a loading control. (E and F) SiHa cells transfected with miR-NC or miR-96 mimics were treated with ropivacaine $(0.5 \mathrm{mM})$ for $72 \mathrm{~h}$, followed by western blot analysis of pSTAT3 levels with tubulin as a loading control (E) and CCK-8 assay (F). Results are shown as means \pm SD of triplicate measurements. The unpaired Student t-test was used to analyze the data. "P<0.05. NS, not significant; pSTAT3, phosphorylated signal transducer and activator of transcription 3; $M E G 2$, maternally expressed gene 2.

examined its effect on the activity of JAK2, a key upstream protein kinase of STAT3 (2). As shown in Fig. 5A, ropivacaine had little effect on JAK2 phosphorylation, suggesting that the drug may instead modulate that of STAT3 via its negative regulators. Protein tyrosine phosphatases (PTPs) regulate the transcriptional activity of STAT3 by dephosphorylating it (18). To study whether PTPs participate in the reduction of STAT3 phosphorylation by ropivacaine, we treated $\mathrm{SiHa}$ cells with $0.5 \mathrm{mM}$ ropivacaine, with or without the PTP inhibitor pervanadate, for $72 \mathrm{~h}$ and then performed western blot analysis. As shown in Fig. 5B, ropivacaine significantly decreased STAT3 phosphorylation, a process that pervanadate completely reversed. Various PTPs, including PTPRD, PTPRT, SHP1, SHP2 and MEG2, have been reported to regulate STAT3 phosphorylation in multiple types of cancers and be involved in carcinogenesis (2). To identify which PTP may mediate the effect of ropivacaine, we first assessed the expression of PTPRD, PTPRT, SHP1, SHP2 and MEG2 in SiHa cells after ropivacaine treatment. The results showed that ropivacaine markedly enhanced MEG2 expression, but we observed no significant changes in the other PTPs (Fig. 5C), suggesting that $M E G 2$ mediates the ropivacaine-induced decrease in STAT3 phosphorylation. To test this possibility, we transiently transfected SiHa cells with MEG2 or normal-control (NC) small interfering RNA (siRNA) and then treated them with $0.5 \mathrm{mM}$ ropivacaine for $72 \mathrm{~h}$. Successful depletion of MEG2 in SiHa cells transfected with MEG2 siRNA (Si-MEG2) was confirmed by real-time PCR analysis and western blot analysis (Fig. 5D and E). MEG2 silencing almost completely rescued the reduction of phosphorylated STAT3 induced by ropivacaine (Fig. 5F). Accordingly, MEG2 knockdown significantly attenuated the suppression of STAT3 transcriptional activation and cervical cancer cell viability mediated by ropivacaine (Fig. 5G). Taken together, these results indicated that MEG2 mediated the inhibitory effect of ropivacaine on phosphorylation of STAT3.

Ropivacaine upregulates MEG2 by suppressing expression of $m i R-96$. We next investigated how ropivacaine enhances $M E G 2$ expression. Recent studies have shown that MEG2 expression in cancers is mainly controlled by microRNAs, including miR-24 (19), miR-96 (20), miR-126 (21), miR-181a-5p (22) and miR-613 (23). Therefore, we began by exploring the effect of ropivacaine on expression of these microRNAs. Interestingly, we found that the drug significantly increased the expression of miR-96 alone, observing no significant changes in 
the other microRNAs (Fig. 6A). This indicated that miR-96 downregulation may lead to a ropivacaine-induced increase in $M E G 2$. To test this, we transiently transfected miR-96 mimic into SiHa cells (Fig. 6B) and then measured the expression of MEG2 after ropivacaine treatment. As shown in Fig. 6C, real-time PCR analysis revealed the successful overexpression of miR-96 after ropivacaine treatment. Furthermore, transfection of miR-96 mimic completely reversed the ropivacaine-induced increase in MEG2 (Fig. 6D); accordingly, transfection also completely abrogated the decrease in STAT3 phosphorylation caused by ropivacaine. Furthermore, transfection of miR-96 mimic markedly attenuated ropivacaine's inhibitory effect on STAT3 phosphorylation (Fig. 6E) and significantly abrogated its suppression of cervical cancer cell growth (Fig. 6F). Collectively, these results indicated that ropivacaine upregulated $M E G 2$ by suppressing expression of miR-96.

\section{Discussion}

Accumulating evidence suggests that local anesthetics play a beneficial role in reducing cancer recurrence (24). Ropivacaine, a widely used local anesthetic, has been shown to suppress the growth of a variety of cancer cells via diverse molecular mechanisms. It can inhibit the growth of breast cancer cells by disrupting mitochondrial function (25), and it has been reported to suppress that of chronic myeloid leukemia by decreasing phosphatidylinositol-4,5-bisphosphate 3-kinase $(\mathrm{PI} 3 \mathrm{~K}) /$ protein kinase $\mathrm{B}(\mathrm{Akt}) /$ mammalian target of rapamycin (mTOR) signaling (12). Ropivacaine has also been found to attenuate gastric cancer cell proliferation via downregulation of extracellular signal-regulated kinase 1/2 (ERK1/2) signaling (15). Despite these reports of the drug's antitumor benefits, its effect on cervical cancer cell growth remains to be elucidated. In the present study, we found that ropivacaine exerted an inhibitory effect on cervical cancer cell growth. Our subsequent experiments demonstrated that its inhibition of cell progression and promotion of cell apoptosis led to suppression of such growth. In contrast with the previously elucidated molecular mechanism underlying the anticancer effect of ropivacaine, we found that ropivacaine attenuated cervical cancer cell growth by suppressing phosphorylation and transcriptional activation of signal transducer and activator of transcription 3 (STAT3). Since elevated levels of phosphorylated STAT3 are frequently found in cervical cancer tissue and are positively correlated with poor prognosis $(5,6)$, our findings may provide novel insight into the treatment of cervical cancer. In addition, as ropivacaine has been reported to suppress migration and invasion of cancer cells in various types of cancers (26-28), and STAT3 activation contributes to migration and invasiveness of cervical cancer (29), it would be important to explore whether ropivacaine affects cervical cancer cell migration or invasion via suppression of STAT3 activation in future investigations.

Phosphorylation and transcriptional activation of STAT3 are precisely modulated by upstream protein kinase and phosphatase (2). Our results indicated that ropivacaine had little effect on the activation of Janus associated kinase 2 (JAK2), the key protein kinase for STAT3, suggesting that the drug may inhibit STAT3 phosphorylation by affecting protein tyrosine phosphatases (PTPs). In accordance with this hypothesis, we found that the suppression of STAT3 phosphorylation mediated by ropivacaine was reversed by the PTP inhibitor pervanadate. Our further experiments demonstrated that maternally expressed gene 2 (MEG2) mediated the suppression of STAT3 phosphorylation induced by ropivacaine. Su et al first showed that $M E G 2$ could dephosphorylate STAT3 at Y705 via their direct interaction in breast cancer cells and that the inactivation of STAT3 by MEG2 decreased the growth of breast tumors (30). Subsequent studies demonstrated that dephosphorylation of STAT3 by MEG2 plays important roles in multiple physiological and pathological processes. Physiologically, MEG2 was reported to regulate erythroid-cell development by dephosphorylating STAT3 (31). Pathologically, MEG2-mediated STAT3 dephosphorylation is involved in the development of breast (30), prostate (32) and colorectal (33) cancers. However, whether the molecular mechanism is the same in the progression of cervical cancer remains unclear. We found that ropivacaine repressed STAT3 phosphorylation and cervical- ancer cell growth by increasing expression of $M E G 2$, which established a functional link between $M E G 2$ and STAT3 phosphorylation in cervical cancer.

In a variety of cancer types, abnormal expression of $M E G 2$ is mainly caused by disordered miRNA expression. Liu et al revealed that $M E G 2$ is negatively regulated by miR-181a-5p and is a tumor-suppressing gene in gastric cancer (22). By targeting MEG2, miR-24 and miR-96 suppress breast cancer cell growth and migration $(19,20)$. In cervical cancer, miR-613 has been shown to contribute to tumor progression by repressing expression of MEG2 (23). Therefore, we speculated that ropivacaine may increase expression of $M E G 2$ by regulating that of these miRNAs. Interestingly, ropivacaine treatment markedly suppressed expression of miR-96 only, having little effect on that of other miRNAs. Consistent with these findings, miR-96 has been reported to be upregulated in human cervical cancer tissues and to function as an oncogene to promote carcinogenesis (34). Apart from cervical cancer, miR-96 has been shown to be involved in various other types of cancers $(35,36)$. Whether ropivacaine exerts a similar anticancer effect on these cancer types deserves further investigation. In addition, the molecular mechanism by which it represses miR-96 expression remains to be clarified in further studies.

In conclusion, our results indicated that ropivacaine inhibited the growth of cervical cancer cells by suppressing the miR613/MEG2/pSTAT3 axis. These results revealed the potent anti-growth effect of ropivacaine in cervical cancer and provide novel insights into the molecular mechanism by which this drug exerts its anticancer activity. Our findings suggest that ropivacaine could have potential use as a novel agent to treat cervical cancer patients.

\section{Acknowledgements}

Not applicable.

\section{Funding}

This research study was supported by the Science and Technology Project of Guangdong Province (2015A020211001), China. 


\section{Availability of data and materials}

The datasets used during the present study are available from the corresponding author upon reasonable request.

\section{Authors' contributions}

$\mathrm{XC}$ ad XS designed the experiments. XC, WL, XG and SH performed the experiments and analyzed the data, $\mathrm{XC}$ and XS wrote the manuscript. All authors read and approved the manuscript and agree to be accountable for all aspects of the research in ensuring that the accuracy or integrity of any part of the work are appropriately investigated and resolved.

\section{Ethics approval and consent to participate}

Not applicable.

\section{Patient consent for publication}

Not applicable.

\section{Competing interests}

The authors declare that they have no competing interests.

\section{References}

1. Shi Y, Zhang Z, Qu X, Zhu X, Zhao L, Wei R, Guo Q, Sun L, Yin X, Zhang Y and Li X: Roles of STAT3 in leukemia (review). Int J Oncol 53: 7-20, 2018.

2. Johnson DE, O'Keefe RA and Grandis JR: Targeting the IL-6/JAK/STAT3 signalling axis in cancer. Nat Rev Clin Oncol 15: 234-248, 2018.

3. Wang Y, Shen Y, Wang S, Shen Q and Zhou X: The role of STAT3 in leading the crosstalk between human cancers and the immune system. Cancer Lett 415: 117-128, 2018.

4. Torre LA, Bray F, Siegel RL, Ferlay J, Lortet-Tieulent J and Jemal A: Global cancer statistics, 2012. CA Cancer J Clin 65: 87-108, 2015.

5. Chen CL, Hsieh FC, Lieblein JC, Brown J, Chan C, Wallace JA, Cheng G, Hall BM and Lin J: Stat3 activation in human endometrial and cervical cancers. Br J Cancer 96: 591-599, 2007.

6. Takemoto S, Ushijima K, Kawano K, Yamaguchi T, Terada A, Fujiyoshi N, Nishio S, Tsuda N, Ijichi M, Kakuma T, et al Expression of activated signal transducer and activator of transcription-3 predicts poor prognosis in cervical squamous-cell carcinoma. Br J Cancer 101: 967-972, 2009.

7. Shukla S, Shishodia G, Mahata S, Hedau S, Pandey A, Bhambhani S, Batra S, Basir SF, Das BC and Bharti AC: Aberrant expression and constitutive activation of STAT3 in cervical carcinogenesis: Implications in high-risk human papillomavirus infection. Mol Cancer 9: 282, 2010.

8. Shukla S, Mahata S, Shishodia G, Pandey A, Tyagi A, Vishnoi K, Basir SF, Das BC and Bharti AC: Functional regulatory role of STAT3 in HPV16-mediated cervical carcinogenesis. PLoS One 8: e67849, 2013.

9. Yong L and Guang B: Intraperitoneal ropivacaine instillation versus no intraperitoneal ropivacaine instillation for laparoscopic cholecystectomy: A systematic review and meta-analysis. Int J Surg 44: 229-243, 2017.

10. de Oliveira GS Jr, Ahmad S, Schink JC, Singh DK, Fitzgerald PC and McCarthy RJ: Intraoperative neuraxial anesthesia but not postoperative neuraxial analgesia is associated with increased relapse-free survival in ovarian cancer patients after primary cytoreductive surgery. Reg Anesth Pain Med 36: 271-277, 2011.

11. Biki B, Mascha E, Moriarty DC, Fitzpatrick JM, Sessler DI and Buggy DJ: Anesthetic technique for radical prostatectomy surgery affects cancer recurrence: A retrospective analysis. Anesthesiology 109: 180-187, 2008.
12. Zheng Q, Peng X and Yu H: Local anesthetic drug inhibits growth and survival in chronic myeloid leukemia through suppressing PI3K/Akt/mTOR. Am J Med Sci 355: 266-273, 2018.

13. Le Gac G, Angenard G, Clément B, Laviolle B, Coulouarn C and Beloeil $\mathrm{H}$ : Local anesthetics inhibit the growth of human hepatocellular carcinoma cells. Anesth Analg 125: 1600-1609, 2017.

14. Bundscherer A, Malsy M, Gebhardt K, Metterlein T, Plank C, Wiese $\mathrm{CH}$, Gruber M and Graf BM: Effects of ropivacaine, bupivacaine and sufentanil in colon and pancreatic cancer cells in vitro. Pharmacol Res 95-96: 126-131, 2015.

15. Yang W, Cai J, Zhang H, Wang G and Jiang W: Effects of lidocaine and ropivacaine on gastric cancer cells through down-regulation of ERK1/2 phosphorylation in vitro. Anticancer Res 38: 6729-6735, 2018.

16. Zhang Y, Peng X and Zheng Q: Ropivacaine inhibits the migration of esophageal cancer cells via sodium-channel-independent but prenylation-dependent inhibition of Rac1/JNK/paxillin/FAK. Biochem Biophys Res Commun 501: 1074-1079, 2018.

17. Wang HW, Wang LY, Jiang L, Tian SM, Zhong TD and Fang XM: Amide-linked local anesthetics induce apoptosis in human non-small cell lung cancer. J Thorac Dis 8: 2748-2757, 2016.

18. Xiong A, Yang Z, Shen Y, Zhou J and Shen Q: Transcription factor STAT3 as a novel molecular target for cancer prevention. Cancers (Basel) 6: 926-957, 2014.

19. Du WW, Fang L, Li M, Yang X, Liang Y, Peng C, Qian W, O'Malley YQ, Askeland RW, Sugg SL, et al: MicroRNA miR-24 enhances tumor invasion and metastasis by targeting PTPN9 and PTPRF to promote EGF signaling. J Cell Sci 126: 1440-1453, 2013.

20. Hong Y, Liang H, Uzair-Ur-Rehman, Wang Y, Zhang W, Zhou Y, Chen S, Yu M, Cui S, Liu M, et al: miR-96 promotes cell proliferation, migration and invasion by targeting PTPN9 in breast cancer. Sci Rep 6: 37421, 2016.

21. Zhu J, Li H, Ma J, Huang H, Qin J and Li Y: PTPN9 promotes cell proliferation and invasion in Eca109 cells and is negatively regulated by microRNA-126. Oncol Lett 14: 1419-1426, 2017.

22. Liu Z, Sun F, Hong Y, Liu Y, Fen M, Yin K, Ge X, Wang F, Chen X and Guan W: MEG2 is regulated by miR-181a-5p and functions as a tumour suppressor gene to suppress the proliferation and migration of gastric cancer cells. Mol Cancer 16: 133, 2017.

23. Li WT, Wang BL, Yang CS, Lang BC and Lin YZ: MiR-613 promotes cell proliferation and invasion in cervical cancer via targeting PTPN9. Eur Rev Med Pharmacol Sci 22: 4107-4114, 2018.

24. Cassinello F, Prieto I, del Olmo M, Rivas S and Strichartz GR: Cancer surgery: How may anesthesia influence outcome? J Clin Anesth 27: 262-272, 2015.

25. Gong X, Dan J, Li F and Wang L: Suppression of mitochondrial respiration with local anesthetic ropivacaine targets breast cancer cells. J Thorac Dis 10: 2804-2812, 2018.

26. Piegeler T, Schläpfer M, Dull RO, Schwartz DE, Borgeat A, Minshall RD and Beck-Schimmer B: Clinically relevant concentrations of lidocaine and ropivacaine inhibit TNF $\alpha$-induced invasion of lung adenocarcinoma cells in vitro by blocking the activation of Akt and focal adhesion kinase. Br J Anaesth 115: 784-791, 2015.

27. Xu YJ, Li SY, Cheng Q, Chen WK, Wang SL, Ren Y and Miao $\mathrm{CH}$ : Effects of anaesthesia on proliferation, invasion and apoptosis of LoVo colon cancer cells in vitro. Anaesthesia 71: 147-154, 2016.

28. Li R, Xiao C, Liu H, Huang Y, Dilger JP and Lin J: Effects of local anesthetics on breast cancer cell viability and migration. BMC Cancer 18: 666, 2018.

29. Fan Z, Cui H, Xu X, Lin Z, Zhang X, Kang L, Han B, Meng J, Yan Z, Yan X and Jiao S: MiR-125a suppresses tumor growth, invasion and metastasis in cervical cancer by targeting STAT3. Oncotarget 6: 25266-25280, 2015.

30. Su F, Ren F, Rong Y, Wang Y, Geng Y, Wang Y, Feng M, Ju Y, Li Y, Zhao ZJ, et al: Protein tyrosine phosphatase Meg2 dephosphorylates signal transducer and activator of transcription 3 and suppresses tumor growth in breast cancer. Breast Cancer Res 14: R38, 2012.

31. Bu Y, Su F, Wang X, Gao H, Lei L, Chang N, Wu Q, Hu K, Zhu X, Chang Z, et al: Protein tyrosine phosphatase PTPN9 regulates erythroid cell development through STAT3 dephosphorylation in zebrafish. J Cell Sci 127: 2761-2770, 2014.

32. Jung SN, Shin DS, Kim HN, Jeon YJ, Yun J, Lee YJ, Kang JS, Han DC and Kwon BM: Sugiol inhibits STAT3 activity via regulation of transketolase and ROS-mediated ERK activation in DU145 prostate carcinoma cells. Biochem Pharmacol 97: 38-50, 2015. 
33. Wang D, Cheng Z, Zhao M, Jiao C, Meng Q, Pan H, Xie Y, Li L, Zhu Y, Wang W, et al: PTPN9 induces cell apoptosis by mitigating the activation of Stat3 and acts as a tumor suppressor in colorectal cancer. Cancer Manag Res 11: 1309-1319, 2019.

34. Ma X, Shi W, Peng L, Qin X and Hui Y: MiR-96 enhances cellular proliferation and tumorigenicity of human cervical carcinoma cells through PTPN9. Saudi J Biol Sci 25: 863-867, 2018.
35. Lin H, Dai T, Xiong H, Zhao X, Chen X, Yu C, Li J, Wang X and Song L: Unregulated miR-96 induces cell proliferation in human breast cancer by downregulating transcriptional factor FOXO3a. PLoS One 5: e15797, 2010.

36. Haflidadóttir BS, Larne O, Martin M, Persson M, Edsjö A, Bjartell A and Ceder Y: Upregulation of miR-96 enhances cellular proliferation of prostate cancer cells through FOXO1. PLoS One 8: e72400, 2013. 